dingungen fänden (da unter solchen Umständen primär an persönlicher Bereicherung interessierte Führungsschichten kein lohnendes Betätigungsfeld mehr vorfänden!).

Weitere Beiträge der Sammlung diskutieren mit vorwiegend rechtsphilosophischem Erkenntnisinteresse Aspekte des Werkes von Karl Christian Friedrich Krause und Christian Wolff (Peter Landau) bzw. Thomas Paine (Simonetta Scandellari, Ferrara) bzw. theoretische Fundierungen des strafrechtlichen Vorsatzbegriffs (Ulrich Schroth). Siegfried Broß entwirft ein Programm zur Bewältigung von "Akzeptanzproblemen" bei staatlichen Entscheidungen, auch er damit ein Thema anschneidend, das die Entwicklungschancen von Rechtsstaatlichkeit in unterschiedlichen kulturellen Kontexten je verschieden betrifft. Broß gibt, gespeist von Erfahrungen hierzulande, dazu eine Art "Fürstenspiegel" (nur geht es nicht um Fürsten, sondern um eine unübersichtliche und diffuse politische Klasse). Ein Beitrag von Lothar Philipps beschließt den Band: "Tu-Tu 2. Von Rechtsbegriffen und neuronalen Netzen" - ein kleines Kabinettstück, das ein rechtstheoretisches Schulbeispiel des dänischen Rechtsphilosophen Alf Ross aus den 50er Jahren mit Einsichten der modernen Computertechnik konfrontiert.

Die Vorträge des Scholler-Symposiums liegen, wie deutlich geworden sein wird, quer zu gewohnten fachlichen Rastern. Gewiß sind sie teils unschwer dem Bereich rechtsphilosophisch inspirierter Strafrechtsforschung oder auch der "reinen" Rechtsphilosophie zuzuordnen. Teilweise inspirieren sie besonders den Interessenten des vergleichenden Verfassungsrechts, den Rechtshistoriker, die rechtswissenschaftliche Entwicklungsländerforschung. Insgesamt aber bereichert der Band das Nachdenken über die Entstehung und die Rolle von Recht sowie seine soziokulturelle Bedingtheit im allgemeinen - wie es sich auch für die Person und das wissenschaftliche Werk des Geehrten sagen läßt.

Philip Kunig

Emmanuel G. Bello / Prince Bola A. Ajibola (eds.)

Essays in Honour of Judge Taslim Olawale Elias

Vol. I: Contemporary International Law and Human Rights

Vol. II: African Law and Comparative Public Law

Martinus Nijhoff Publishers, Dordrecht/Boston/London, 1992, XXVIII, 898 p., US\$ 265.00

Taslim O. Elias war einer der international bekanntesten Juristen der zweiten Hälfte des Jahrhunderts, gewiß der bekannteste aus Afrika. Seine internationale Präsenz begann zu Beginn der 60er Jahre und kulminierte in der Wahl zum Mitglied des Internationalen Gerichtshofs im Jahre 1976. Von 1982 bis 1985 war Elias dessen Präsident, gehörte dem Gericht dann noch weitere sechs Jahre an. Geboren 1914, aufgewachsen in Lagos, akademisch ausgebildet in London, galt das Interesse Elias' zunächst dem traditionellen nigeria- 
nischen Recht. Sogleich mit der Unabhängigkeit Nigerias (1960) gelangte Elias in höchste Ämter, war zunächst Justizminister, wurde in dieser Eigenschaft 1961 Generalberichterstatter der ersten African Conference on the Rule of Law, welche die International Commission of Jurists (Genf) in Lagos veranstaltete - eine Konferenz, die (entgegen vielen anderen zum gleichen Thema) erwähnenswert ist, weil sie während einer wichtigen Phase der Entkolonisierung afrikanische Juristen aus britisch und französisch geprägten Erlebniswelten zusammenbrachte und den Ruf nach Rechtsstaatlichkeit und Menschenrechtsschutz vernehmlich erhob. Erst 25 Jahre später trat die Afrikanische Konvention der Menschenund Volksrechte (Banjul Charta) in Kraft. Schon 1963 war die Organisation der Afrikanischen Einheit gegründet worden; manche glaubten damals, solche "Einheit" sei herstellbar, dies mit unterschiedlichen Konzepten; 30 Jahre nach Gründung der OAU stehen US-Amerikaner im UNO-Auftrag in Somalia, um das Land vor dem Versinken zu bewahren und um massenhaftem Sterben Einhalt zu gebieten. Andere Interventionen gingen voraus, weitere werden gewiß folgen.

Vom afrikanischen Elend, im einzelnen wie im besonderen, handeln auch die vorliegenden Bände, genauer gesagt: Sie lassen dieses Elend durchscheinen, allerdings aus festlichem Anlaß. Zurück daher noch einmal zur Person des Geehrten, auch deshalb, weil diese Festschrift ein sehr persönliches Werk ist: Sie ist nicht ein Sammelband aus Anlaß eines Geburtstages, sie nimmt vielmehr grosso modo den Geehrten zum Anlaß, über Fragen zu publizieren, welche im Zentrum seines Wirkens gestanden haben.

T.O. Elias entstammt dem im internationalen Konzert über die Zeit hin gewichtigsten schwarzafrikanischen Staat. Seinen Stuhl im Internationalen Gerichtshof nimmt nun erneut ein Nigerianer ein, der Mitherausgeber dieser Festschrift, Ajibola. Glänzend in London vorbereitet, fiel Elias die Rolle des prägenden nigerianischen Juristen zu, nicht nur durch wissenschaftliche Aktivität, sondern auch durch politische Gestaltung, zunehmend auch auf internationalen Bühnen, bald auch im Sinne einer Schulenbildung; Elias ist der Förderer der meisten später zu Einfluß gelangten nigerianischen Rechtslehrer gewesen. Er erlangte Funktionen und Ehren in einer Vielzahl, wie es keinem anderen afrikanischen Juristen bisher beschieden war. Wissenschaftlich ist er - nach gründlichen Studien der nigerianischen Rechtsentwicklung, aber auch zu weiteren westafrikanischen Rechtsordnungen, vor allem in den 50er und 60er Jahren hervorgetreten. Später hat er sich zunehmend als Völkerrechtler geäußert. Sein "Africa and the Development of International Law" (1972), auch die "New Horizons" (1979), haben in Europa und Amerika Bekanntheit erlangt, sein Versuch, die Rolle der Generalversammlung der Vereinten Nationen und ihre Entschließungen in Analogien zu parlamentarischen Strukturen erfassen zu wollen (wohl erstmals in den Essays in Honour of Philip C. Jessup, hrsg. 1972 von Friedmann Henkin und Lissitzyn, vorgetragen), wird gerade in europäischen Schriften oft vermerkt, allerdings durchgängig verworfen.

Spiritus rector der vorliegenden Festschrift ist Emmanuel G. Bello, ein gleichfalls in Europa, vor allem in England, Frankreich, auch in Deutschland bekannter nigerianischer Gelehrter (etwa: "African Customary Humanitarian Law", 1980). Professor Bello wirkt 
jetzt als Direktor einer Judge Elias Research Foundation in Lagos. Er schreibt im Vorwort, daß die Autoren der Festschrift für eine Balance stünden zwischen "older distinguished masters in the science of law from the industrialized and sophisticated world, and the younger generation of international legal brains from the developing nations". Es sind dies insgesamt 44 Autoren, darunter - was augenfällig ist - 18 Nigerianer, aus Afrika ferner der für die afrikanische Menschenrechtsdiskussion prägende Senegalese Kéba M'Baye, auch der mittlerweile zum Generalsekretär der Vereinten Nationen ernannte Boutros Ghali, und dazu klangvolle Namen überwiegend der älteren Generation, vor allem aus den USA und Großbritannien (wie - z.B. - Bin Cheng, James E. Fawcett, Louis Henkin, Michael Reisman, Oscar Schachter, Georg Schwarzenberger), schließlich Kollegen Elias' aus den Reihen des Internationalen Gerichtshofs oder der International Law Commission, wie Roberto Ago, Manfred Lachs, Nagendra Singh. Französische Wissenschaft wird repräsentiert durch Prosper Weil, die deutschsprachige durch Stephan Verosta und Ignaz Seidl-Hohenveldern.

Die Beiträge sind geordnet in sieben Kapitel. Auf einen einleitenden essay von Ian Brownlie über das Völkerrecht in der zweiten Hälfte des 20. Jahrhunderts folgt ein allgemeiner Abschnitt zu Grundfragen des Völkerrechts (13 Beiträge), ein solcher zum Internationalen Gerichtshof (3), zu den Menschenrechten (11), zum afrikanischen Gewohnheitsrecht (2), zum nigerianischen Recht (4), zum "Verfassungsrecht" (4), davon drei erneut Nigeria betreffende Beiträge, schließlich sieben Miscellanea. Der Gesamtinhalt der Festschrift betrifft damit das Interessenfeld dieser Zeitschrift, zu den einzelnen Themata müssen hier einige Hinweise genügen. So greift Reisman die Soft Law-Frage auf, bündig seine Position zusammenfassend, Seidl-Hohenveldern reflektiert über die bekannte Entscheidung des Bundesgerichtshofs zu den nigerianischen Kunstschätzen, dieses grundsätzlich, aber auch im Lichte neuerer Entwicklungen, McWhinney äußert sich zur ethno-kulturellen und "Rechtskreis"-bezogenen Zusammensetzung des Internationalen Gerichtshofs. Besonderes Interesse verdient die menschenrechtliche Abteilung der Festschrift, die überwiegend Fragen des Menschenrechtsschutzes im Blick auf die Bedingungen ungleicher Entwicklung, speziell auch in Afrika und zu den Vorschriften der Banjul Charta erörtert. Teilweise ergiebig wird der Problematik der Rechte der Völker nachgegangen. Der Herausgeber Bello selbst bietet eine längere Studie zu Art. 22 der Banjul Charta, dies in Auseinandersetzung auch mit mancher in Europa zu der Konstruierbarkeit, der Funktion und dem Sinn des "Right to Development" geäußerten Auffassung.

Die Festschrift für Elias ist eine editorische Leistung. Sie bringt afrikanische, vor allem nigerianische Stimmen zu Völkerrechtsfragen und solchen des innerstaatlichen Rechts wieder einmal nachhaltig zu Gehör und zeigt sie im Dialog mit vor allem der anglo-amerikanischen Wissenschaft. Sie dokumentiert die Lebensleistung Elias', indem sie die Probleme thematisiert, um deren Bewältigung dieser in verschiedenen juristischen Rollen über Jahrzehnte gerungen hat. Sie gibt vielen einflußreichen und hochrangigen Weggefährten des Geehrten Gelegenheit, ihm Reverenz zu erweisen. Die Festschrift belegt aber auch Zustände: Sie macht, über festschriftübliche Emphasen noch hinaus und bis in viele Einzelbeiträge hinein sichtbar, wie individuell geprägt, wie personalorientiert die Community der 
Rechtsgelehrten und Praktiker erscheint, sobald der Blick sich über Amerika und Europa hinaus richtet. $\mathrm{Da}$ ein einzelner als gleichsam für sich allein stehender, höchster wissenschaftlicher Repräsentant eines ganzen kontinents erscheint, indiziert den Rückschluß auf die Wissenschaft dieses Kontinents im übrigen; künftig und für die nächste Generation der afrikanischen Rechtswissenschaft wird das, so läßt sich schon heute sagen, nicht mehr möglich oder nötig sein. Auch dazu hat Elias übrigens beigetragen. Ferner: Ein derartiges Werk tatsächlich (und nicht nur, wie hier, dem Anspruch nach) gesamtafrikanisch oder sogar gesamthaft auf die Dritte Welt bezogen zu gestalten, würde wohl derzeit nicht gelingen können; nicht "Africa and International Law" stehen hier vor uns, sondern, was die afrikanischen Beiträge anlangt, ein im wesentlichen die nigerianische Wissenschaft repräsentierendes Werk. Auch das gibt Anlaß zum Nachdenken. Und schließlich inhaltlich: Vieles, was hier thematisch auf der Agenda steht, treibt Völkerrechtspraxis und Rechtswissenschaft seit Jahren um, manches seit Jahrzehnten. Das gilt für Grundsatzfragen wie gerade auch für den Menschenrechtsschutz im besonderen, speziell auch für das Right to Development. "We must now bring this study to an end", sagt Bello (S. 472) nach eingehenden Uberlegungen und Analysen zum extensiven Schrifttum, und (in der Schlußpassage seines Beitrages): "To take Article 22 of the Charta seriously as a legal principle ... is no easy matter", "in the not too distant future" sollten "regional integration and cooperation for the purposes of development" erreicht sein "without pain and without tears". Mit allen diesen Bemerkungen hat Bello Recht. Die Verhältnisse sind nicht so, daß sie bereits auf allgemeine Bemerkungen dieser Art verzichten ließen.

Philip Kunig

\section{Hans Hattenhauer}

Europäische Rechtsgeschichte

C.F. Müller, Heidelberg 1992, 825 S., DM 164,--

$\mathrm{Ab}$ und an erscheint ein Werk, das für rechtshistorisch Interessierte eine wahre Freude ist. Das galt seinerzeit für Wilhelm Grewes "Epochen der Völkerrechtsgeschichte" (BadenBaden 1984, Studienausgabe 1988). Das gilt, bezogen auf die Wissenschaftsgeschichte eines Teilgebiets der Jurisprudenz, für Michael Stolleis' jüngst um den zweiten Band ergänzte "Geschichte des öffentlichen Rechts in Deutschland" (München 1988 und 1992). Und das gilt schließlich auch für das hier angezeigte Buch von Hans Hattenhauer zur europäischen Rechtsgeschichte. Es ist ein in jeder Hinsicht umfassendes Werk, behandelt es doch die Geschichte im Prinzip aller Rechtsgebiete, über den gesamten Zeitraum vom Recht der archaischen Kulturen bis hin zur Gegenwart und geographisch für Gesamteuropa samt seiner "Auswirkungen" auf den Rest der Welt. 